\title{
Collisional Stripping of Mercury's Mantle
}

\author{
WILLY BENZ, ${ }^{*}$ WAYNE L. SLATTERY, $\dagger$ AND A. G. W. CAMERON* \\ *Harvard-Smithsonian Center for Astrophysics, Harvard College Observatory, 60 Garden Street, \\ Cambridge, Massachusetts 02138, and $\dagger$ Los Alamos National Laboratory, Los Alamos, New Mexico 87545
}

Received June 19, 1987; revised October 7, 1987

\begin{abstract}
We investigated the conditions under which a giant collision between a hypothetical proto-Mercury and a planet one-sixth its mass would result in the loss of most of the silicate mantle of the planet, leaving behind an iron-rich planet and thus explaining the anomalously high density of Mercury. We carried out a series of numerical simulations using our three-dimensional smoothed particle hydrocode, varying the impact parameter and the relative velocity between the planet and impactor. We demonstrate that the details of the equation of state do not play an important role. We show that a head-on collision at $20 \mathrm{~km} / \mathrm{sec}$ and an off-axis (impact parameter equal to half the radius of proto-Mercury) collision at $35 \mathrm{~km} / \mathrm{sec}$ are about equivalent as far as damage to protoMercury is concerned. Both collisions leave behind a remnant that has the required characteristics of the present Mercury. Whether this scenario is actually successful depends on the size of the condensates in the ejected cloud of debris. Preliminary estimates show that most of the ejected mass is probably removed from Mercury-crossing orbits. If this turns out to be true, a giant collision is a plausible explanation for the strange density of Mercury. O 1988 Academic Press, Inc.
\end{abstract}

\section{INTRODUCTION}

The mean density of Mercury is anomalously high, $5.44 \mathrm{~g} / \mathrm{cm}^{3}$, or, removing the effects of compression, $-5.3 \mathrm{~g} / \mathrm{cm}^{3}$ (Smith 1979). From this, Urey $(1951,1952)$ noted that Mercury must have an iron-to-silicate ratio much larger than that for any other terrestrial planet. The iron-to-silicate mass ratio has been estimated to lie in the range $66: 34$ to $70: 30$, about twice that of any of the other terrestrial planets, the Moon, or the Eucrite Parent Body (Basaltic Volcanism Study Project 1981). For comparison note that the mean density of the Earth is $5.52 \mathrm{~g} / \mathrm{cm}^{3}$, corresponding to an uncompressed density of $\sim 4.45 \mathrm{~g} / \mathrm{cm}^{3}$ (Lewis 1972).

A variety of hypotheses have been suggested to account for the strange density of Mercury. A particularly promising one is the suggestion that one or more major collisions with Mercury blew off the bulk of the silicate mantle from the original Mercury protoplanet (Smith 1979, Wetherill 1985). Since the escape velocity from Mercury is rather low and relative velocities between colliding planets at Mercury's orbit are high, one can reasonably expect that a major collision might indeed result in an appreciable loss of material. What is less evident, however, is whether one can achieve a selective loss of material, in which the silicate mantle is preferentially lost relative to the iron core.

In this paper we investigate the possibility of this selective loss of material using our program of three-dimensional hydrodynamic calculations of planetary collisions to simulate the effects of single collisions upon a Mercury protoplanet. For the hypothetical protoplanet we take an estimated mass of the present iron core in Mercury and restore enough missing silicates to give the planet approximately chondritic composition. This leads to a protoplanetary 
mass some 2.25 times the present mass of Mercury. Wetherill (1986) has noted that major terrestrial protoplanets are quite likely to have had collisions with planetary bodies of comparable or only slightly inferior mass. He has also noted that in the region of formation of Mercury the expected random velocities are sufficiently high that a collision of proto-Mercury with a comparable mass is likely to be completely disruptive. For these reasons we chose to subject our Mercury protoplanet to collisions with an object one-sixth its mass. We have varied the collision velocity and the impact parameter in the set of runs reported here. We did not vary the mass of the impactor. One has to bear in mind that the only real constraint we have in this case is the observed mean density, which by itself is not sufficient to determine the parameters of the collision. We present these results in order to impart some flavor of the types of results to be expected in such collisions.

\section{SMOOTHED PARTICLE HYDRODYNAMICS}

The calculations reported here made use of a three-dimensional hydrodynamic code run on Cray X-MP supercomputers at the Los Alamos National Laboratory. The method used is called "smoothed particle hydrodynamics"; this method has been described in detail by Benz et al. (1986), where it was used to investigate the origin of the Moon as a result of a major planetary impact.

The "smoothed particle hydrodynamics" (SPH) method was first introduced by Lucy (1977) and was shown to be especially powerful in many applications, mainly by the work of Gingold and Monaghan (1982, 1983) and more recently by Monaghan (1986). Since this method has been described many times elsewhere, we only recall briefly its general principles; a reader interested in the details should consult the above references. SPH is a free Lagrangian approach for solving the conservation equations of hydrodynamics. A finite set of spatially extended particles replaces the continuum of hydrodynamic variables. The usual mesh is not needed; this technique therefore does not suffer from mesh tangling or inaccuracies associated with severe distortion of the mesh. The method is therefore particularly suited for the simulation of highly distorted flows such as those occurring during impacts. The simulation evolves in time by computing the trajectories of all particles, given the various forces acting between them. These forces are computed from the interactions between the particles that are derived from the particles' relative positions and velocities.

The three-dimensional shape of a particle is given by a function called the "kernel." Although the primary use of this function is to allow interpolation, it may be viewed as the density distribution of an individual particle. Several kernels have been proposed in the literature-polynomials (Lucy 1977), Gaussians (Gingold and Monaghan 1982, 1983), and exponentials (Wood 1981, Benz et al. 1986). Recently, however, Monaghan and Lattanzio (1985) have proposed the use of splines defined on a compact support. Since their method not only allows good interpolations but also speeds up the computation, we adopted their kernel $\left(W_{4}\right.$ in their notation) in these calculations.

For calculations described by Benz et al. (1986, see also Benz et al. 1987), a modified Mie-Gruneisen equation of state of the Tillotson (1962) form was used. With this equation of state, granite was chosen to represent rocky material, since the relevant data were available at Los Alamos. We also used the Tillotson granite equation of state for the first seven of our proto-Mercury runs reported here. Beyond our Mercury run 7 we used the ANEOS equation of state which was developed at the Sandia Laboratories (Thompson and Lauson 1984). This analytical equation of state includes treatment of the melt, liquid-vapor, and solidvapor transitions. Each phase is described in terms of its Helmholtz free energy potential. Separate phases are treated as separate 
components in a mixture that is always in pressure and temperature equilibrium. Mixed phases can coexist at the same Gibbs potential. We used dunite to represent rocky material with this equation of state; the appropriate equation-of-state parameters for dunite were determined by Jay Melosh, and we are grateful to him for providing them to us. The main reason for the change was that the Tillotson equation of state does not give a thermodynamically consistent description of a two-phase medium and therefore may lead to errors in determining pressure gradients. The ANEOS equation treats mixed phases in a thermodynamically correct manner. It appears, however, that the sensitivity of the results to the equation of state is small (see detailed results below). This observation is encouraging since any known equation of state is stretched beyond testable range in the kind of situation we are simulating, and too large a dependence on its details would result in large uncertainties in the final results.

For the first two proto-Mercury collision runs we used a relatively small number of particles; these results were for our preliminary orientation to the problem, and we do not report them here. Runs 3 and 4 had more particles and used rock plus an iron core for the target (amounting to just less than a third of its mass) but just rock for the projectile. After run 4 the projectile had an iron core as well. In these runs the protoMercury target was represented by 3000 particles, of which 959 were iron and 2041 were silicate rock. The projectile of onesixth the mass was represented by 1000 particles, each half the mass of the particles used in the target. In the runs in which the projectile had an iron core (beyond run 4) 319 of the particles were iron and 681 were rock.

In the smoothed particle method, if two particles are far away from each other, they interact by gravitational attraction only, and pressure gradients are negligible or zero. After the collision has taken place and the debris has spread out in an expanding cloud into space we reach a situation where the density of particles is so low that they only interact gravitationally. From there on, a hydrodynamical treatment is no longer necessary and the system can be treated as a standard $N$-body problem to be solved on a personal computer. Such a debris cloud is made of single particles as well as of clumps of particles. We solved the $N$ body problem for those clumps containing more than three particles. Each clump was first replaced by one particle having the total mass of the clump and located at its center of mass. These larger clumps were followed in their motions under their mutual gravitational forces (neglecting forces due to the Sun and the other planets) until it could be determined whether they had escaped from the site of the collision, or until they fell back and merged together into a central object. The smaller clumps (of three particles or less, mostly single particles) were individually followed in their trajectories until they (predominantly) escaped or were reaccumulated. The results listed in the tables are those obtained at the end of these additional calculations.

\section{CENTRAL COLLISIONS}

A summary of the results of our collision calculations is shown in Table I. In run 3 the projectile had an impact velocity of 27 $\mathrm{km} / \mathrm{sec}$ at infinity; it hit the target centrally and knocked off most of the silicate rock and some of the iron. This would be a good candidate for the kind of collision that would produce the observed Mercury, except that the composition of the projectile is unrealistic (the projectile was made of rock exclusively). In run 4 the velocity of the impactor was increased to $38 \mathrm{~km} / \mathrm{sec}$, resulting in complete disruption of the target.

With run 5 we started a realistic series of cases by putting an iron core into the projectile (its mass again just below a third of the planet's mass). The impact velocity was $25 \mathrm{~km} / \mathrm{sec}$ and the target was once again totally disintegrated. The denser core in 
TABLE I

The Outcome of the Mercury Collision Scenarios

\begin{tabular}{|c|c|c|c|c|c|c|c|c|}
\hline Case & $\begin{array}{l}\text { Velocity } \\
(\mathrm{km} / \mathrm{sec})\end{array}$ & $\begin{array}{c}\text { Impact } \\
\text { parameter }\end{array}$ & $\begin{array}{l}\text { Target } \\
\text { iron }\end{array}$ & $\begin{array}{l}\text { Projectile } \\
\text { iron }\end{array}$ & $\begin{array}{l}\text { Target } \\
\text { silicate }\end{array}$ & $\begin{array}{l}\text { Projectile } \\
\text { silicate }\end{array}$ & $\begin{array}{l}\text { Silicate/iron } \\
\text { ratio }\end{array}$ & $\begin{array}{l}\text { Mass } \\
\text { ratio }\end{array}$ \\
\hline 3 & 27 & 0.00 & \multirow{2}{*}{\multicolumn{2}{|c|}{$76.5 \%$}} & $5.1 \%$ & $0.4 \%$ & \multirow[t]{2}{*}{0.14} & \multirow[t]{2}{*}{0.63} \\
\hline 4 & 38 & 0.00 & & & Disir & tegrated & & \\
\hline \multicolumn{9}{|c|}{ Below here the impactor had an iron core } \\
\hline 5 & 25 & 0.00 & \multicolumn{6}{|c|}{ Disintegrated } \\
\hline 6 & 20 & 0.00 & $56.1 \%$ & $37.7 \%$ & $5.9 \%$ & $7.5 \%$ & 0.25 & 0.56 \\
\hline 7 & 22 & 0.46 & $93.1 \%$ & $7.2 \%$ & $22.0 \%$ & $2.8 \%$ & 0.51 & 1.02 \\
\hline \multicolumn{9}{|c|}{ Below here the ANEOS equation of state was used } \\
\hline 8 & 20 & 0.00 & $48.8 \%$ & $42.0 \%$ & $8.8 \%$ & $11.3 \%$ & 0.41 & 0.57 \\
\hline 9 & 15 & 0.00 & $86.2 \%$ & $69.3 \%$ & $42.1 \%$ & $31.3 \%$ & 1.03 & 1.43 \\
\hline 10 & 10 & 0.00 & $99.2 \%$ & $97.8 \%$ & $79.1 \%$ & $58.6 \%$ & 1.64 & 2.19 \\
\hline 11 & 15 & 0.59 & $99.4 \%$ & $0.6 \%$ & $70.3 \%$ & $12.3 \%$ & 1.55 & 1.82 \\
\hline 12 & 20 & 0.51 & $94.3 \%$ & $3.8 \%$ & $47.1 \%$ & $6.8 \%$ & 1.08 & 1.42 \\
\hline 13 & 28 & 0.53 & $80.0 \%$ & $0.0 \%$ & $20.0 \%$ & $1.2 \%$ & 0.54 & 0.89 \\
\hline 14 & 35 & 0.54 & $57.0 \%$ & $0.3 \%$ & $7.8 \%$ & $0.3 \%$ & 0.29 & 0.53 \\
\hline
\end{tabular}

Note. The impact parameter is given in units of the target radius. The values given for target and projectile iron and silicate are the percentages of the numbers of particles in the residual body coming from these four sources. The mass ratio is the residual mass relative to the present mass of the planet Mercury $\left(3.3 \times 10^{26} \mathrm{~g}\right)$.

this impactor enabled the target to be disintegrated at a significantly lower velocity than in the softer case in which the impactor consisted only of rock. In run 6 the velocity was lowered to $20 \mathrm{~km} / \mathrm{sec}$ and the target survived the collision, after once again losing nearly all its silicates. It is interesting to note, however, that the iron core in the target lost $43.9 \%$ of its mass but picked up $37.7 \%$ of the mass of the iron core in the projectile. This is an excellent candidate to be the scenario for the formation of Mercury.

The ANEOS equation of state was used in run 8 and in subsequent runs. As stated above, this equation of state provides a much better treatment of mixed-phase regimes. The importance of this on the outcome of the collision was questioned in our simulations about the origin of the Moon (see a somewhat more detailed discussion of this point in Benz et al. 1987). Therefore the first case we ran using this equation of state, run 8 , was a repeat of the conditions in run 6 . The results of these two runs were very similar, indicating that the results do not depend very sensitively on the character of the equation of state or on the choice of rocky material. The amount of iron left in the residual body is nearly the same; there is a little less iron from the target and a little more from the projectile. The total mass left behind after the collision is nearly the same in the two cases. This mass amounts to $60 \%$ of the present mass of Mercury, which provides an opportunity for Mercury to reaccumulate some of the mass blown away in the collision which remains in solar orbits crossing the orbit of Mercury.

Runs 9 and 10 were head-on collisions like run 8 , but at progressively lower collision velocities, $15 \mathrm{~km} / \mathrm{sec}$ for run 9 and 10 $\mathrm{km} / \mathrm{sec}$ for run 10 . The amount of mass ejected in the collision decreases with decreasing collision velocity, so that in the 10 $\mathrm{km} / \mathrm{sec}$ case the planet is left with about the mass it had at the beginning. Neither of these cases is a candidate to produce the current planet Mercury.

We noticed some striking features of 
these head-on collisions. As an illustration we show in Fig. 1 snapshots of the evolution of run 8 . This run $(20 \mathrm{~km} / \mathrm{sec}$ relative velocity and zero impact parameter) is what we called above a successful case, since the collision left less than a Mercury mass with the appropriate iron-to-silicate ratio. At the time the two planets hit, a very strong jet- ting occurs in a plane almost perpendicular to the collision axis since this is the direction of least resistance (Fig. 1b). By the time the shock has reached the boundaries one sees ejecta flying away downrange as well as uprange (Figs. 1c, d). As the collision proceeds (Fig. ld) more and more material is jetted out and the two cores are

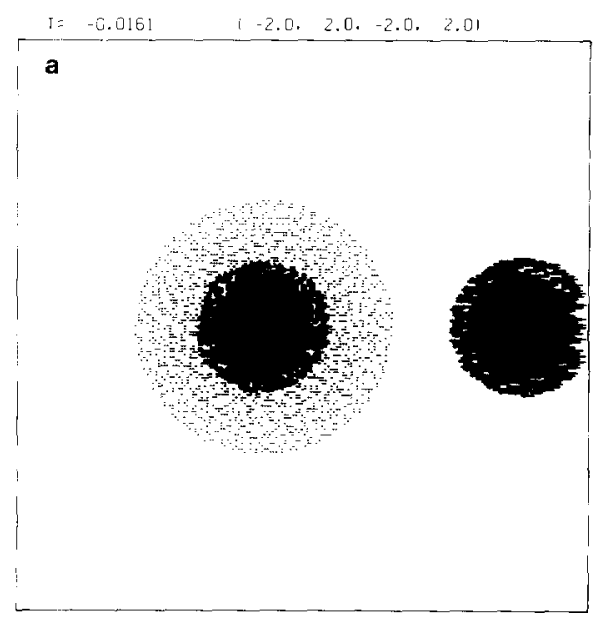

$0.464 E+01$ MAXIMUM VECTOR

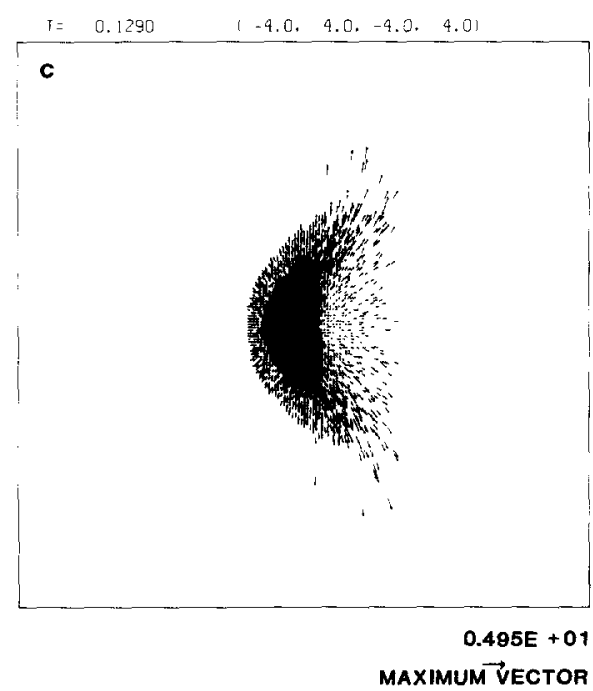

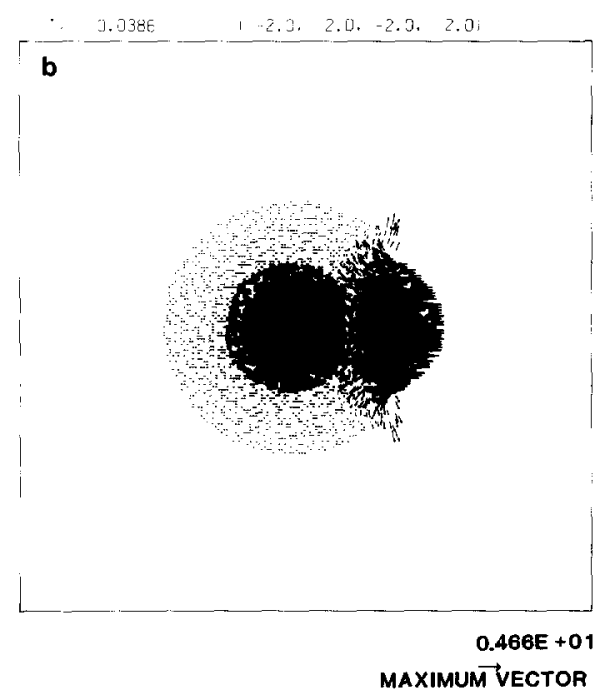

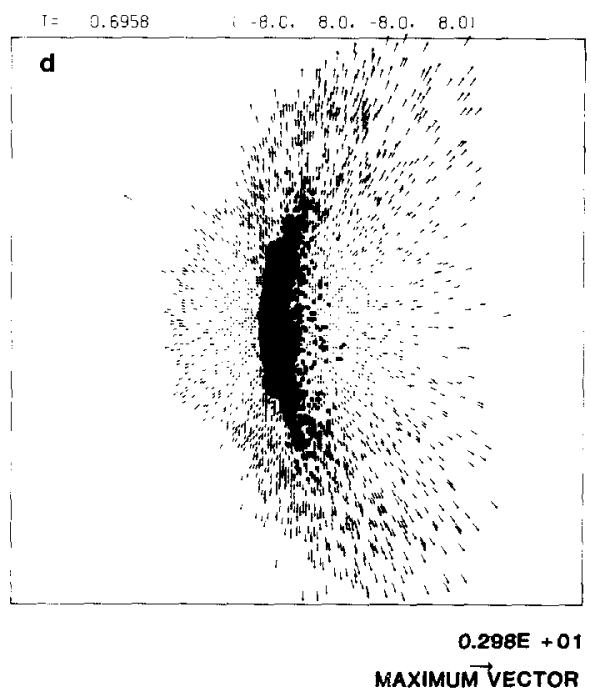

FIG. 1. Snapshots taken during the evolution of run $8\left(v_{\text {inf }}=20 \mathrm{~km} / \mathrm{sec}\right.$, head-on). Velocity vectors are plotted at particle locations. The velocity has been normalized to its maximum value in each frame. Time (in hours from first impact) and coordinates of the four corners of the plotted field (in units of $3.46 \times 10^{8} \mathrm{~cm}$ ) are given in the upper line (note the change of scale between frames). For iron particles, a $\bullet$ is plotted at the bottom of the arrow. 

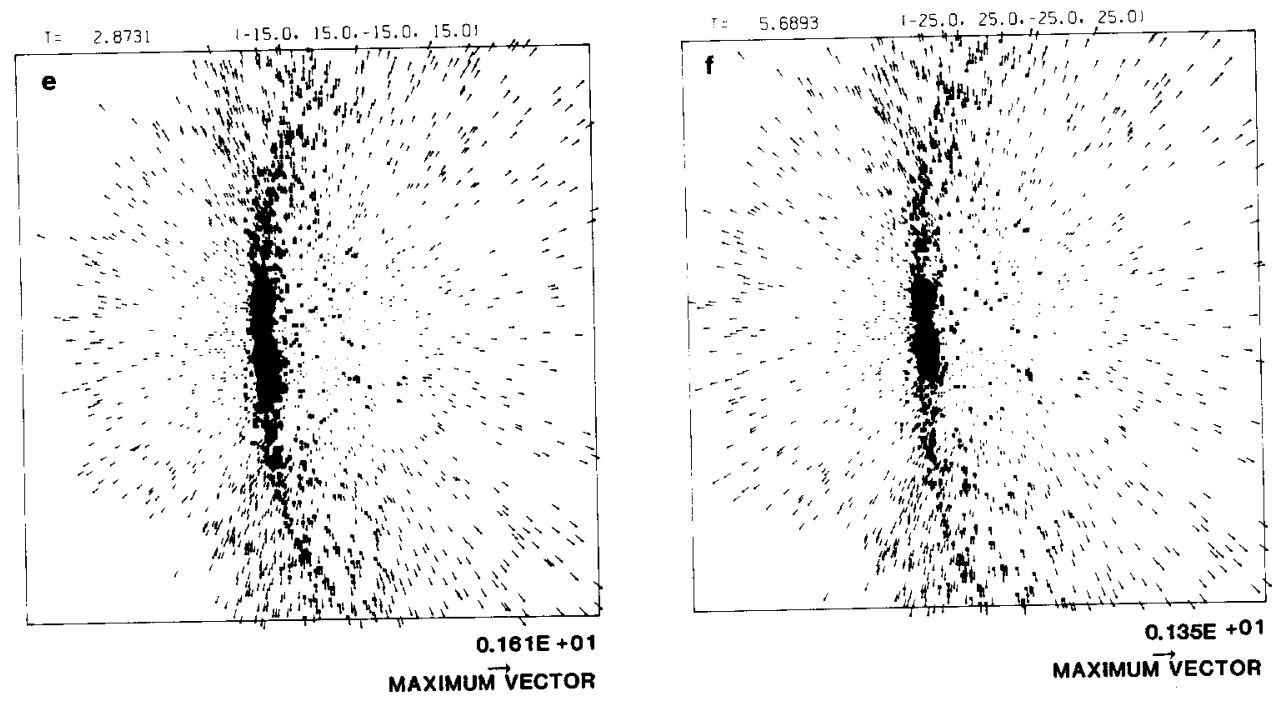

FIG. 1-Continued.

transformed into a thin sheet lying in the plane perpendicular to the collision axis (Fig. 1d). This sheet, made mostly of iron, is the slowest expanding material. The expansion velocity is low enough to prohibit this material from escaping its own gravitational attraction. The inner parts of the "plate" eventually break up into a few clumps (Fig. 1e). The somewhat remarkable distribution of these clumps is best seen if one looks at the system along the collision axis. Figure 2 displays for four different times the evolution of the central region after the collision and the formation of an annulus. The clumps forming this annulus (almost entirely iron), together with some surrounding material, are still gravitationally bound and eventually fall back (there is no angular momentum present since the collision was head-on), collapsing into a final (spherical) object whose characteristics are listed in Table I. Due to computing time limitations we could not follow the evolution that far with the SPH code; the hydrodynamic simulations stopped after the equivalent of a little bit more than $5 \frac{1}{2}$ hr of real time (Fig. 1f). Beyond that point we followed the system with a personal computer in the way described above.
At the lower collision velocities, the formation of this iron annulus was not so clear; the core never really disrupts but rather collapses from the "plate-like" configuration to the form of a stubby cigar with its long axis in the direction of the collision, before relaxing into a more rounded shape. At the higher collision velocities the whole target planet would break into fragments spreading apart in the plane at right angles to the direction of the collision, in a way similar to that illustrated in Fig. 1. The expansion velocities of the fragment are now high enough so they manage to escape their own gravitational attraction, resulting in complete destruction of the planet.

\section{OFF-AXIS COLLISIONS}

Runs 7 and 11 and higher runs involved cases in which the projectile hit the target off-center, with an impact parameter around half the radius of the target. The impact parameter given in Table $I$ is in fact the distance of closest approach that the planets would have if they were point masses. The striking characteristic of these runs turned out to be that the off-center collision is much less effective in ejecting mass from the target. This is easily understand- 


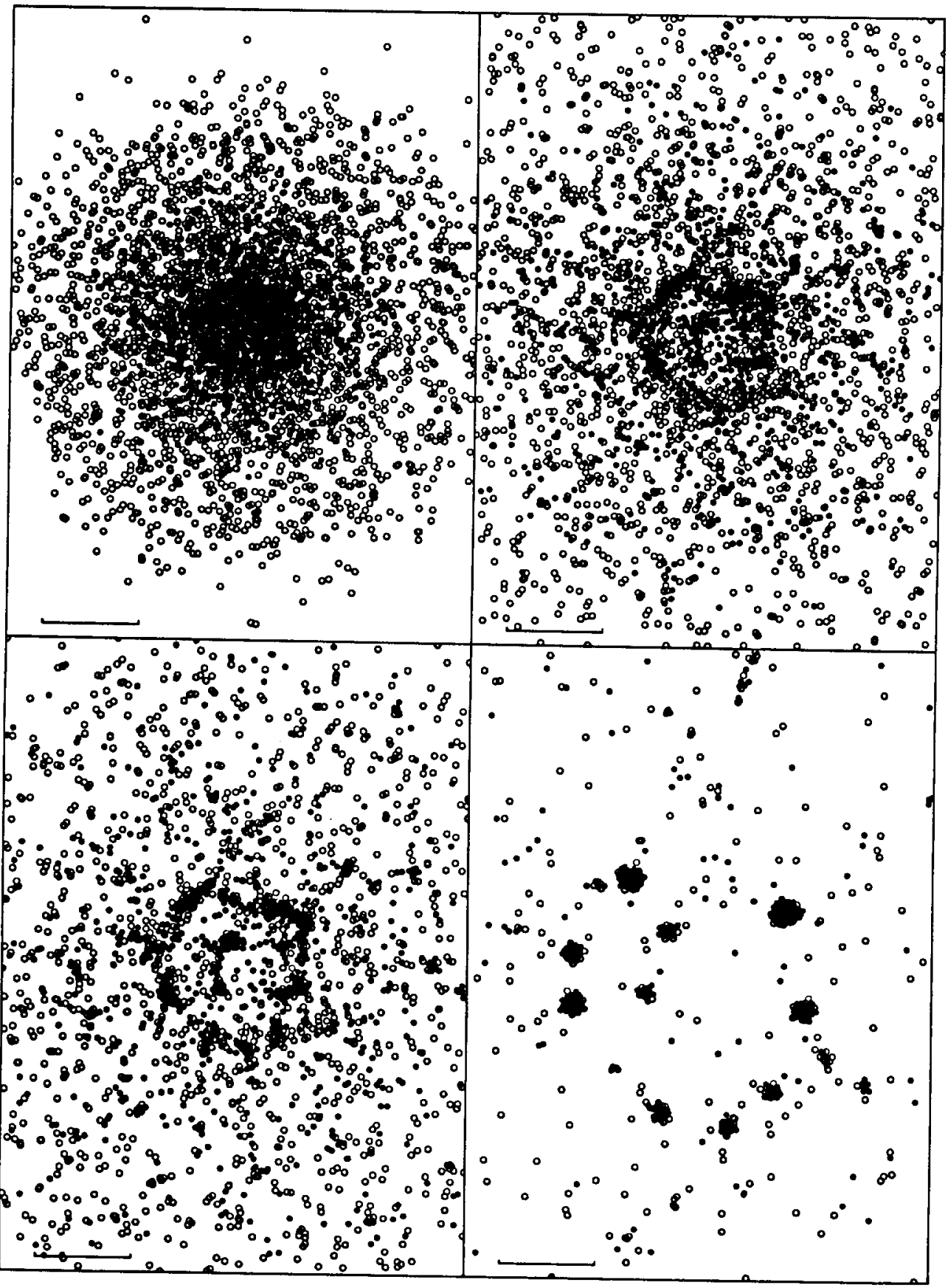

FIG. 2. Plots of the positions of the silicate (open circles) and iron (filled circles) particles at four different times taken from Cray run 8 , viewed from the direction of collision. The bar shown at the bottom of each plot represents a distance of $10^{9} \mathrm{~cm}$. After the first panel, only the central region of the mass distribution is contained within the boundaries of the plot. 
able since jetting mechanisms responsible for ejecting mass strongly depend on the strength of the shock, which is maximized for a head-on collision. Whereas a central collision at $20 \mathrm{~km} / \mathrm{sec}$ leaves a residue of only 0.57 Mercury mass, a similar collision that is noncentral leaves 1.42 Mercury mass, obviously too large a mass to make this a candidate collision to have produced the present Mercury. Only in run 14, where the impact velocity was $35 \mathrm{~km} / \mathrm{sec}$, does the planetary remnant become reduced to 0.53 Mercury mass, which makes it a good candidate for forming the observed planet. Thus this off-axis collision at $35 \mathrm{~km} / \mathrm{sec}$ is equivalent to a central collision of $20 \mathrm{~km} /$ sec in its destructive effects. It is clear that the collision velocity required to produce this degree of destruction is a steep function of the impact parameter.

The evolution of this collision is illustrated in a series of snapshots in Fig. 3.
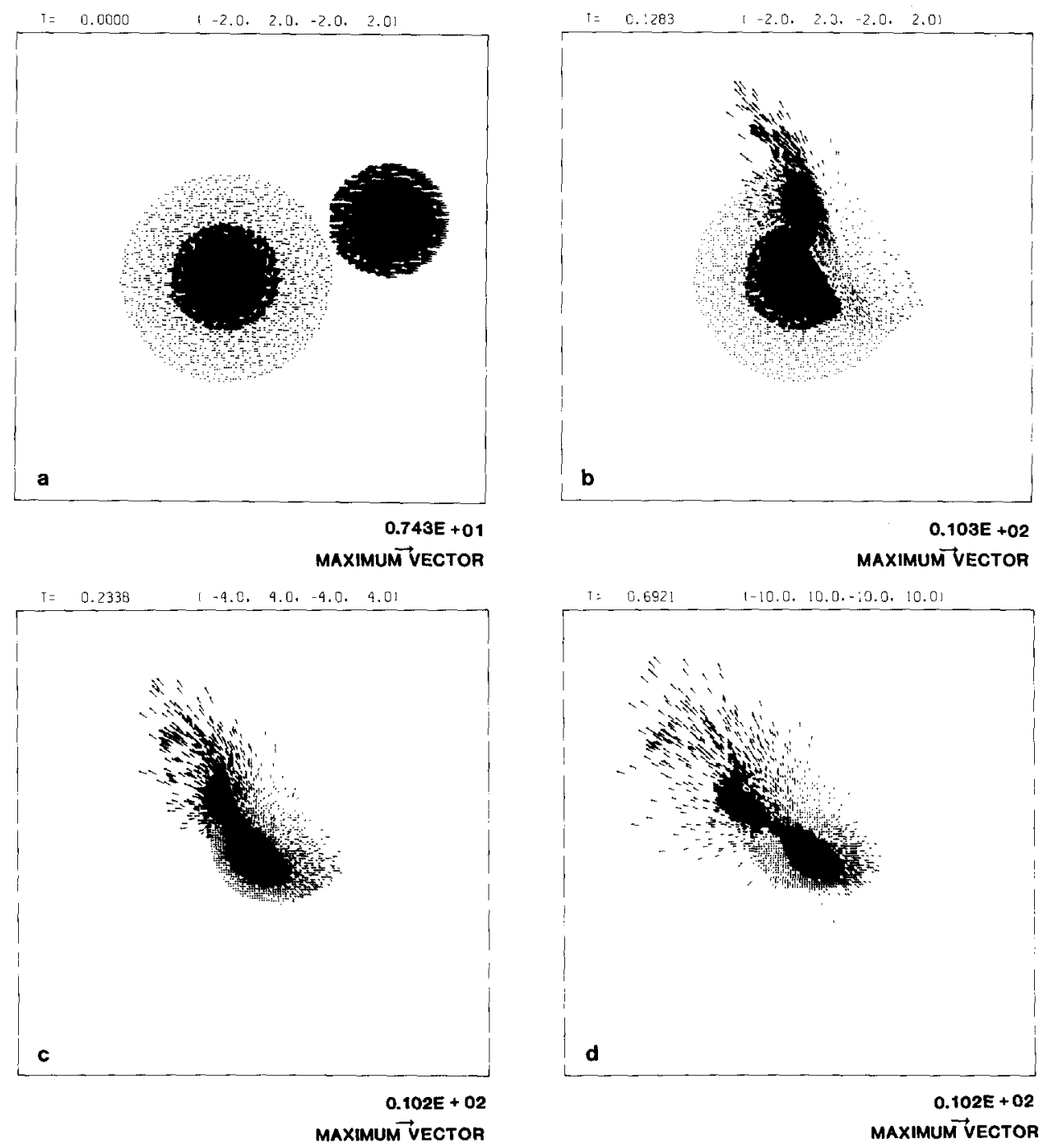

FIG. 3. Snapshots taken during the evolution of run $14\left(v_{\text {inf }}=35 \mathrm{~km} / \mathrm{sec}\right.$, off-axis). The symbols have the same meaning as in Fig. 1. 

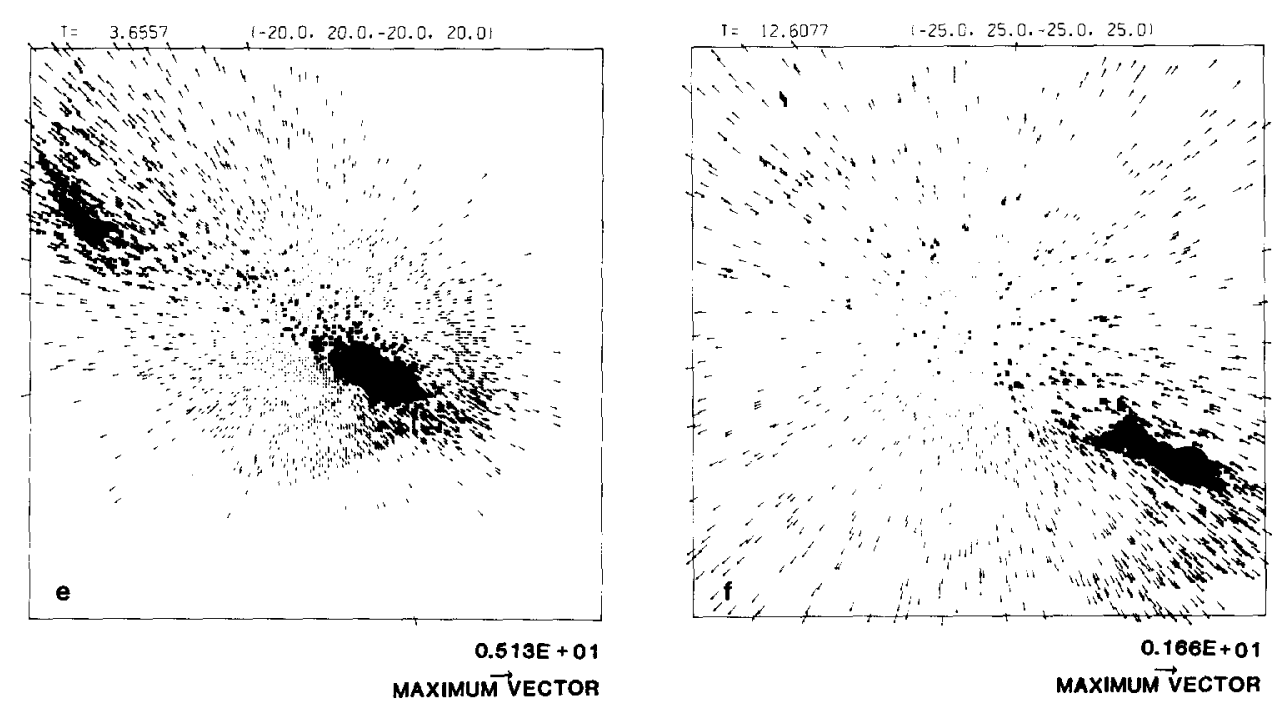

FIG. 3-Continued.

Again, one sees strong jets during early times (Fig. 3a) followed by almost complete destruction of both planets. A remarkable feature of these plots is the selective way the shock affects the silicate mantle and the iron core. Figures $3 \mathrm{e}$ and $\mathrm{f}$ give the impression that the mantle literally explodes and separates from the core which seems to suffer somewhat less from the collision. This is the key mechanism that really allows the separation of the mantle from the core and makes the giant impact scenario a plausible one to explain the strange density of Mercury.

\section{ACCEPTABLE CONDITIONS}

As discussed earlier, the present silicate/ iron ratio in Mercury is about 0.4-0.5. As we shall see, subsequent reaccumulation is more likely to add silicates to the collision remnant that iron (mainly because only a little iron was ejected), and hence the silicate/iron ratio should generally increase during the course of subsequent accretion. Thus, for a remnant produced in these calculations to be considered a candidate protoplanet leading to the present Mercury, the silicate/iron ratio following the collision should be less than 0.5. As may be seen in
Table 1 , only the central collisions near 20 $\mathrm{km} / \mathrm{sec}$ and the noncentral collisions near $35 \mathrm{~km} / \mathrm{sec}$ satisfy this criterion.

A separate but related criterion is that the collision remnant should have a total mass less than that of Mercury now. This condition would be weakened if Mercury were to sustain one or more further destructive collisions of large magnitude.

We conclude therefore that the only cases in which we have found conditions leading to nearly complete silicate loss and relatively little net iron loss from protoMercury are a nearly central collision at about $20 \mathrm{~km} / \mathrm{sec}$ and a noncentral collision at about $35 \mathrm{~km} / \mathrm{sec}$. The amount of mass is a sensitive function of the collision velocity and the impact parameter.

\section{FATE OF EJECTED MATTER}

In the course of a typical collision a great deal of material is thrown out from the site of the collision, some to escape and the rest to fall back onto the planet. Of the material thrown out, some is clumped into clusters of particles and the rest consists of single particles. The larger clumps are composed predominantly of iron; the single particles are almost entirely made of rock. During 
the course of the collision itself, the great majority of these single silicate particles are in a condition which the ANEOS equation of state describes as vaporized or mixed phase, partly vapor and partly condensed liquid. Upon expansion of the material, cooling will occur, so that at a later time the particles may show as totally in a condensed phase. Of course, the resolution of our calculation and the input physics does not allow the determination of the number and sizes of the condensations.

Raizer (1960) made an estimate of the size of condensations in a cloud of vaporized matter expanding in a vacuum. Although he was concerned with an iron meteorite striking the Moon, which is far from this problem, his results may give us an indication of what should be expected. Detailed calculations of this point are planned in a later paper since this is a rather critical point that determines largely what happens to the ejected matter. Raizer found that about half the iron escapes into space in the form of gas that has not condensed, and the other half condenses into particles of radius roughly $10^{-5} \mathrm{~cm}$. The theory of Raizer also predicts that the sizes of the condensates should scale with the dimension of the region in which condensation takes place and depend to some power of the inverse of the initial energy input. The conclusion one is tempted to draw from this is that most of the ejected matter from the proto-Mercury collisions condenses (if at all) in very small particles.

There is an additional complication in the present case. The condensation of iron is a direct process, since the compositions of the gas and condensed phases are the same. But rock vapors are decomposed into simpler molecules in the vapor phase, so that when these molecules land on the surface of a condensate, they must wander around the surface looking for their complementary molecules in order to form a molecule of the condensed phase, and they must succeed before evaporating off the surface. The effects of this on the nucleation and growth of the particles must be evaluated. We plan to investigate this situation in more detail.

If most of the material ejected in the collision were to be reaccumulated upon Mercury, then the collision would have made only a minor perturbation to the composition of the planet. Thus the collision can produce the strange composition of Mercury only if a majority of the ejected silicate fraction does not reaccumulate upon the planet. To prevent reaccumulation of this material by Mercury it has to be removed from Mercury-crossing orbits. There are at least two major effects that can achieve this for the condensed particles: perturbation by other planets and the Poynting-Robertson effect. We will give some consideration to the two mechanisms below. Individual gas molecules that have not condensed will quickly become ionized and will then be carried away by the solar wind.

\section{Perturbation by Other Planets}

We have considered a "successful" collision to be one in which the mass of the protoplanet is reduced well below that of the present planet Mercury and in which the remnant is composed predominantly of iron. All of the material that is ejected in the collision goes into independent orbit around the Sun; these orbits must necessarily cross the orbit of the protoplanet remnant. The present orbital elements of Mercury are subject to secular variation with time due to other planetary perturbations, so we do not know what the orbit of the remnant would have been, nor do we know the degree to which the orbit would be modified by subsequent collisions of smaller but still substantial magnitude. Nevertheless, to gain some insight into the problem, we took the present orbit of Mercury as a suitable prototype of the remnant orbit, and calculated whether any of the particles ejected from the collision would have been put into orbits that would cross the orbit of Venus. We found that in general only a few of the particles would do so if ejected as 
aphelion, and usually a few dozen and at most a few hundred would do so at perihelion. Thus only a minority of the particles ejected in the collision can be removed from Mercury-crossing orbits by other planetary perturbations.

\section{Poynting-Robertson Effect}

Particles with dimensions of the order of $10^{-5}$ may have optical properties in which solar radiation is strongly absorbed or scattered. In this case the radiation pressure force on the particle may exceed the gravitational attraction of the Sun, so that the particle will be quickly expelled from the Solar System. We consider here particles for which this would not happen.

Because particles reemit solar photons randomly, they lose angular momentum and therefore eventually spiral into smaller and smaller orbits about the Sun. The importance of this effect is dependent on the size of the particles. The important question is to determine the maximum size of a particle that would be removed from a Mercury-crossing orbit in a time shorter than its expected collision time with the planet, which is about $10^{6}$ years (George Wetherill, private communication). We found this size to be of the order of $1 \mathrm{~cm}$. As noted above, a large part of the ejected silicate material will have passed through the vapor phase. Upon expansion, this material will cool and condense into solid particles, but if the vapor is by that time at fairly low density, then the particle sizes will probably be very small, in the subcentimeter size range. Such small particles will be drawn into the Sun by the Poynting-Robertson effect. The remaining question, for which we do not obtain information from our simulation, is what mass fraction of the ejected material is likely to be in form of very small (subcentimeter) particles.

Besides the fact that the particles formed from condensing gas are likely to be small, collisions between these particles will act as a "grinding" mechanism, reducing their sizes even more (if the collision occurs at a high velocity). It is therefore of interest to estimate the time scale between collisions and to compare this time scale again with the collision time scale of a fragment with Mercury. The collision time scale $\tau_{\text {col }}$ can be estimated by

$$
\tau_{\mathrm{col}}=\frac{1}{n \sigma v}
$$

where $n$ is the number density of particles, $\sigma=4 \pi r^{2}$ is the geometrical cross section (the fragments are too small for gravitational focusing to be of any importance), and $v$ is the average random velocity of the fragments. The number density $n$ of fragments is given by $n=M / m_{f} V$, where $M$ is the ejected mass, $m_{f}$ is the mass of one fragment (we have supposed that the total mass is distributed in fragments with a single value of the mass), and $V$ is the volume in which the fragments are dispersed. We estimate this volume by $V=2 \pi\left(R_{\text {out }}^{2}-R_{\text {in }}^{2}\right) h$. In this formula $R_{\text {out }}$ and $R_{\text {in }}$ are, respectively, the outer and inner edges of the disk formed by the fragments and $h$ is half the thickness of this disk. We estimate $h$ in the usual way by $h=\left(v / v_{\text {circ }}\right) R_{\text {mean }}$, with $\boldsymbol{R}_{\text {mean }}$ $=\left(R_{\text {out }}+R_{\text {in }}\right) / 2$ and $v_{\text {circ }}$ the Keplerian velocity at $R_{\text {mean }}$. Inserting these expressions into the equation giving the time scale yields, after some trivial algebra,

$$
\tau_{\mathrm{col}}=\frac{2 \pi \rho r\left(R_{\text {out }}^{2}-R_{\mathrm{in}}^{2}\right) R_{\text {mean }}^{3 / 2}}{3 M \sqrt{G M_{\mathrm{Sun}}}}
$$

Interestingly, the time scale does not depend directly on the random velocities. This comes from the fact that increasing the random velocities increases the thickness of the disk and therefore decreases the number density of fragments, canceling out the velocity dependence of the collisional time scale. Of course, velocity dependence is hidden in the values of the outer and inner boundaries of the disk.

To obtain a numerical estimation for this time scale we introduce the following values: $R_{\text {out }}=0.723 \mathrm{AU}$ (orbit of Venus), $R_{\text {in }}=$ 
$0.19 \mathrm{AU}$ (half the orbit of Mercury), $\rho=3.3$ $\mathrm{g} / \mathrm{cm}^{3}$ (dunite), and $M=6.7 \times 10^{26} \mathrm{~g}$ (results of runs 8 and 14). We obtain $\tau_{\text {col }}=$ $0.18 r$ years ( $r$ in centimeters). The radius of a fragment for which the time between a collision with another fragment is equal to the collision time with Mercury $\left(10^{6}\right.$ years $)$ is therefore $r=56 \mathrm{~km}$. We conclude that collisions among the fragments will be very frequent.

The above estimate assumes that the relative velocities of the particles is random. Initially the particles ejected from the site of the collision are nearly comoving if they are close to one another. However, the orbits of the particles will bring them back to the vicinity of the original collision, at which time proto-Mercury is unlikely to be present. At that time they will have relative velocities comparable to those in the initial ejection, provided they started in dissimilar orbits. The velocities of ejection ranged from nearly zero (particles barely escaping) to about $10 \mathrm{~km} / \mathrm{sec}$, with a few particles being ejected at velocities up to $20 \mathrm{~km} / \mathrm{sec}$.

These collisions are likely to be destructive since they would commonly occur with velocities comparable to or greater than the speed of sound in rock $(\sim 4 \mathrm{~km} / \mathrm{sec})$. This will greatly increase the mass that can be removed from Mercury-crossing orbits by the Poynting-Robertson effect.

\section{CONCLUSIONS}

We have carried out a series of threedimensional numerical simulations of collisions between a proto-Mercury and an object one-sixth its mass. We have shown that for a given impact parameter and a given velocity it is quite possible to remove most of the planet's mantle, leaving behind an object that has about the right iron-to-silicate ratio. The amount of ejected mass decreases very strongly with increasing impact parameter. It is possible to compensate for this by increasing the impact velocity. To have about the same destructive effects as a $20 \mathrm{~km} / \mathrm{sec}$ head-on collision, one needs a velocity of about $35 \mathrm{~km} / \mathrm{sec}$ if the impact parameter is equal to half the radius of the planet. This already high value suggests that the type of collision that produced the present Mercury (if the collisional origin is correct) was close to head-on.

We have investigated elsewhere (Benz et al. 1986, 1987) the collisional origin of the Earth's Moon and have found that this scenario is quite plausible. A criticism that often appears about giant impacts is the low probability that the right collisional parameters (relative velocity, impact parameter, masses, etc.) would occur to produce the observed results. Of course, other collisional parameters would produce large numbers of other unobserved results, which might nevertheless appear quite anomalous. Some set of collisional parameters will be selected by chance, and from this point of view the parameters that appear to be involved in the Moon and Mercury collisional cases do not appear unusual. Several other planets in the Solar System appear to have characteristics that may have been produced predominantly by a single giant collision. Further investigations of these cases are needed.

We note that although in our simulations many collisions have left observational signatures, we have found many more that do not appear to modify the planet in a significant way. Therefore, the existence of apparently "normal" planets (no anomalous density or collisionally produced satellites) is not unexpected. Mars, Venus, and Mercury have no large satellites. Mars, Venus, and the Earth show little deviation in the silicate-to-iron ratio. Venus and Mars are too massive for a collision to eject a significant fraction of their mantle (see Benz et al. 1986, 1987). In the Mars case the planet is only about twice as massive as Mercury, but it is located much further out in the potential well of the Sun. We expect the average collision to occur at too low a speed to produce significant mass low during the collision. 


\section{ACKNOWLEDGMENTS}

We thank Jay Melosh not only for providing us with the ANEOS dunite equation of state, but also for helpful discussions on the conditions for condensation of the ejecta gas. This research has been supported in part by NASA Grant NGR 22-007-269 at Harvard University. W. Benz also acknowledges support from the Swiss National Science Foundation. We are grateful to the Los Alamos National Laboratory for the provision of the computer time that has been used on this problem.

\section{REFERENCES}

Basaltic Volcanism Study Project 1981. Basaltic Volcanism on the Terrestrial Planets. Pergamon Elmsford, NY

Benz, W., W. L. Slattery, and A. G. W. Cameron 1986. The origin of the Moon and the single-impact hypothesis I. Icarus 66, 515-535.

Benz, W., W. L. Slattery, and A. G. W. Cameron 1987. The origin of the Moon and the single-impact hypothesis H. Icarus 71, 30-45.

Gingold, R. A., AND J. J. Monaghan 1982. Kernel estimates as a basis for general particle methods in hydrodynamics. J. Comput. Phys. 46, 429-453.

Gingold, R. A., And J. J. Monaghan 1983. On the fragmentation of differentially rotating clouds. Mon. Not. R. Astron. Soc. 204, 715-733.

LEwIS, J. S. 1972. Metal/silicate fractionation in the solar system. Earth Planet. Sci. Lett. 15, 286-290.

LuCY, L. 1977. A numerical approach to the testing of the fission hypothesis. Astron. J. 82, 1013-1024.
Monaghan, J. J. 1986. Particle method for hydrodynamics. Comput. Phys. Commun. 3, 72-124.

Monaghan, J. J., and J. C. LatTanzio 1985. A refined particle method for astrophysical problems. Astron. Astrophys. 149, 135-143.

RaIzEr, YU. P. 1960. Condensation of a cloud of vaporized matter expanding in vacuum. Sov. Phys. JETP 10, 1229-1235.

Smith, J. V. 1979. Mineralogy of the planets: A voyage in space and time. Mineral. Mag. 43, 1-89.

Thompson, S. L., AND H. S. LAUSON 1984. Improvement in the Chart $D$ Radiation-Hydrodynamic Code. III. Revised Analytic Equations of State. Sandia Laboratories Report SC-RR-71 0714.

Tillotson, J. H. 1962. Metallic Equations of State for Hypervelocity Impacts. General Atomic Report GA-3216, July 1962.

UREY, H. C. 1951. The origin and development of the Earth and other terrestrial planets. Geochim. Cosmochim. Acta 1, 209-277.

UREY, H. C. 1952. The Planets. Yale Univ. Press, New Haven, Conn.

Wetherill, G. W. 1985. Remark at Lunar and Planetary Science Conference, Houston.

Wetherill, G. W. 1986. Accumulation of the terrestrial planets and implications concerning lunar origin. In The Origin of the Moon (W. K. Hartmann, R. J. Phillips, and G. J. Taylor, Eds.). Lunar and Planetary Institute, Houston.

Wood, D. 1981. Collapse and fragmentation of isothermal gas clouds. Mon. Not. R. Astron. Soc. 194, 201-218. 